\title{
İnsansız Hava Araçlarında Gömülü Sistem Üzerinden Derin Öğrenme ile Nesne Tespiti
}

\author{
Ziya Sayg1l1 ${ }^{*}$, Güzin Özmen² \\ ${ }^{1 *}$ Selçuk Üniversitesi, Fen Bilimleri Enstitüsü, Mekatronik Mühendisliği Bölümü, Konya, Türkiye, (ORCID: 0000-0003-3282-0946), ziyasaygili@gmail.com \\ ${ }^{2}$ Selçuk Üniversitesi, Teknoloji Fakültesi, Biyomedikal Mühendisliği Bölümü, Konya, Türkiye (ORCID: 0000-0003-3007-5807), gozmen@selcuk.edu.tr
}

(2nd International Conference on Applied Engineering and Natural Sciences ICAENS 2022, March 10-13, 2022)

(DOI: 10.31590/ejosat.1081713)

ATIF/REFERENCE: Saygılı, Z. \& Özmen, G. (2022). İnsansız Hava Araçlarında Gömülü Sistem Üzerinden Derin Öğrenme ile Nesne Tespiti. Avrupa Bilim ve Teknoloji Dergisi, (34), 292-298.

\begin{abstract}
Öz
Hava robotiği olarak adlandırılan insansız hava araçları (IHHA), afet yönetimi, trafik yoğunluğu ve sınır güvenliği gibi sivil ve askeri alanlarda veri ve görüntü toplamak için son zamanlarda sıklıkla kullanılmaktadır. Kamera görüş açısının değişkenliğinden dolayı İHA ile yüksek irtifada alınan görüntüler üzerinden anlık nesne tespiti yapmanın zorlukları vardır. Bu çalışma İHA' ya CSI (Camera Serial Interface) modülü ile bağlanmış bir kameradan, farklı açı ve koşullarda alınan görseller ile Evrişimli Sinir Ağı tabanlı SSD MobileNet kütüphanesi kullanılarak nesne tespit etmeyi amaçlamaktadır. İHA üzerinden kamera ile elde edilen görüntüler NVIDIA Jetson Nano gömülü sistem bilgisayarı ile işlenmiş ve sınıflandırılmıştır. Gerçek zamanlı hedef tespitinde karşılaşılan problemlerin başında, değişken hava koşulları ve aydınlatma sebebiyle düşük çözünürlüklü elde edilen hareketli nesnelerin sınıflandırılması yer almaktadır. Bu duruma derin öğrenme kütüphanesinde yer alan öğrenilmiş görsellerle, kameradan alınan görseller arasındaki yakın özellikli veriler eşleştirilerek çözüm aranmaktadır. Bu çalışmada gömülü sistem içerisinde bir algoritma oluşturularak elde edilen görüntüler üzerinde düzenleme yapılmış, derin öğrenme kütüphanesi ile özellik karşılaştırma işleminin ardından elde edilen çıktılar nesne sınırı ve mAP (mean Average Precision) ortalama hassasiyet yüzdesi kullanılarak değerlendirilmiştir. Sonuç olarak, İHA üzerinden alınmış gerçek zamanlı görsel verilerden insan tespiti için \%95.5 mAP son kesinlik ve \%69.45 mAP ortalama hassasiyet, araç tespiti için \%83.4 mAP son kesinlik ve $\%$ 64.5 mAP ortalama hassasiyet elde edilmiştir.
\end{abstract}

Anahtar Kelimeler: İHA, Nesne Tespiti, Derin Öğrenme, Gömülü Sistem, SSD MobileNet

\section{Object Detection with Deep Learning using Embedded System in Unmanned Aerial Vehicles}

\begin{abstract}
Unmanned aerial vehicles (UAVs), called aerial robotics, have been frequently used recently to collect data and images in civilian and military areas such as disaster management, traffic density and border security. Due to the variability of the camera view angle, there are difficulties in making instant object detection on the images taken at high altitude with the UAV. This study aims to detect objects from a camera connected to the UAV with the CSI (Camera Serial Interface) module using images taken at different angles and conditions and a Convolutional Neural Network-based SSD MobileNet library. The images obtained with the camera via the UAV were processed and classified with the NVIDIA Jetson Nano embedded system computer. One of the problems encountered in real-time target detection is the classification of moving objects with low resolution due to variable weather conditions and lighting. A solution to this situation is sought by matching the close-featured data between the learned images contained in the deep learning library and the images from the camera. In this study, the images obtained by creating an algorithm in the embedded system were edited, and the outputs obtained after the feature comparison process with the deep learning library were evaluated using the object boundary and mAP (mean Average Precision) average precision percentage. As a result, 95.5\% mAP final accuracy and 69.45\% Map average accuracy for human detection, $83.4 \%$ mAP final accuracy and 64.5\% mAP average accuracy for vehicle detection were obtained from real-time visual data received from the UAV.
\end{abstract}

Keywords: UAV, Object Detection, Deep Learning, Embedded System, SSD MobileNet

* Sorumlu Yazar: ziyasaygili@gmail.com 


\section{Giriş}

Günümüzde derin öğrenme tabanlı nesne tespiti çalışmaları büyük ilgi görmektedir. Sabit kameralar ile kısıtlı açılarda görüntü almanın yetersiz kaldığ 1 durumlarda, birden fazla nesnenin tespit edilmesi gerektiğinde ve büyük ölçekli görüntü verilerinin toplanmasına ihtiyaç duyulduğunda İnsansız hava araçları (İHA) tercih edilmektedir. İHA endüstrisinin hem askeri hem de sivil operasyonlarda geniş bir uygulama yelpazesi bulunmaktadır [1]. Pistsiz kalkış ve iniş, hedefe yönelik operasyon ve geniş çalışma alanı gibi etkinlik avantajları ile en yaygın kullanılan İHA tipi olan çok rotorlu İHA' lar [2], askeri görevler, afet kurtarma, eğimli fotoğraf görüntüleme, tarımsal uygulamalar, lojistik ulaşım, keşif [3], şehirdeki araçların belirlenmesi [4], orman yangınlarının belirlenmesi [5], sınır güvenliği [6], kaçakçılıkla mücadele [7], tehlike tespiti [8], hassas tarım [9] gibi alanlarda fayda sağlamaktadır.

Günümüzde görüntü tabanlı sistemler, ortam modellemesi, insan eylemine bağlı olay tanıma, hedef ve nesne algılama üzerine çalışmalar yapılmaktadır. Aynı zamanda İHA' lara dayalı sivil ve askeri faaliyetlerin desteklenmesiyle birlikte insan-drone iş birliğinde yeni bir dönem başlamaktadır [10].

Nesne tanıma, video veya fotoğraflardaki verilerin tanımlanmasına yarayan bilgisayarla görme olarak da anılan bir uygulamadır. Kameralar ile elde edilen görsel verilerdeki nesnelerin varlığını belirlemek ve sınıflandırmak için çeşitli yöntemler bulunmaktadır. Bu yöntemlerden bazıları YOLO (You Only Look Once), Dense MobileNet, RetinaNet, SSD-MobileNet (Single Shot Detector), R-CNN (Region with Convolutional Neural Networks), Faster R-CNN ve TensorFlow olarak bilinmektedir.

Yapılan literatür araştırmasına göre, Piao ve arkadaşları (2020) çalışmalarında İHA üzerinden almış oldukları video görüntüleriyle YOLO (You Only Look Once) yöntemini kullanarak insan tespiti işlemi yapmışlardır. Sonuçlarını ortalama hassasiyet birimi olan mAP (mean Average Precision) ile değerlendirmişler ve insan için \%68.86 mAP elde etmişlerdir [11]. Yu ve ark. (2021) çalışmalarında, VisionDrone ile araçların dağılımını gösteren yoğunluk haritası oluşturmuşlardır. Böylece araçların o bölgeye dahil olup olmadığını DMNet veri seti ile tespit etmişlerdir. Sonuç olarak araçlarda \%51.7 ortalama hassasiyet (mAP) elde etmişlerdir[12]. Donahue ve ark. (2014) DeCAF (Deep Convolutional Activation Feature) yöntemi ile sabit bir görsel üzerinden hayvan tanıma işlemi yapmış, hayvanların nesne etiketli veri tabanından faydalanarak \% 40.94 ortalama hassasiyet (mAP) elde etmişlerdir [13]. Ngiam ve ark. (2019) LIDAR teknolojisine katkı sağlamak için LIDAR' in görsel haritalarından aldıkları yaya görüntülerini StarNet kütüphanesinde değerlendirmişlerdir. Kullandıkları bu kütüphane ile yaya tespitinde \%66.8 ortalama hassasiyet (mAP) sonucunu ortaya koymuşlardır [14]. Ouyang ve ark. (2015) ise almış oldukları görsellerdeki dairesel nesnelerin görüntü işlemeden sonra kayıplara ve deformasyonlara uğradığını fark etmişılerdir. Önermiş oldukları DeepID-Net kütüphanesi ile nesne tespit analizlerinde golf topu ve teker gibi dairesel nesnelerde \%50.7 ortalama hassasiyet (mAP) elde etmişlerdir [15].

$\mathrm{Bu}$ çalışmada nesne tespiti gerçekleştirmek için yüksek mobilite eğilimi ve coğrafi kısıtlama olmaksızın video verilerinin biriktirebilmesi özelliklerinden dolayı İHA tercih edilmiş ve kamera ile elde edilen anlık görsellerden gömülü sistem üzerinden Evrişimli Sinir Ağları (CNN) yöntemi ile sınır belirleme işlemi gerçekleştirilmiştir. Gömülü sistem ve kütüphanelerinde
CNN'nin yeterli katman ve düğüm noktasının olmaması derin öğrenme yöntemlerinde hareketli hedef/nesne tanıma için yetersiz kalmaktadır [16]. Bu amaçla, bu çalışmada CNN katman ve düğüm noktasının düzenlenebileceği Nvidia Jetson gömülü sistem bilgisayarı kullanılmış, içerisine nesne tespiti ve sınıflandırmada başarılı olan SSD MobileNet Kütüphanesi eklenmiştir. Elde edilen sonuçlar mAP (mean Average Precision) ortalama hassasiyet ile değerlendirilmiştir.

Şekil 1'de Jetson Nano Derin Öğrenme Kütüphane Performanslarının kıyaslanması görülmektedir.

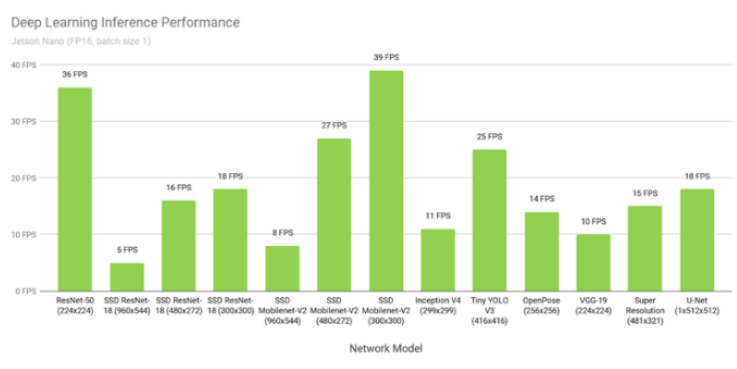

Şekil 1. Jetson Nano Derin Öğrenme Kütüphane Performansları [17]

Şekil 1'de görüldüğü gibi SSD MobileNET 39 fps görüntü performansı ile diğerlerinden avantajlı olması sebebiyle bu çalışmada tercih edilmiştir.

\section{Materyal ve Metot}

\section{1. İnsansız Hava Aracı}

İHA üzerinden nesne tespiti yapılmasının en büyük avantajı, sabit kameraların aksine yüksek hareket kabiliyetine sahip olması ve geniş gözetim yapabilmesidir.

Bu çalışmada kullanılan ve Şekil 2' de görülen İHA' da uçuş kontrolünü sağlayan 6 eksenli bir gyro, manometre ve IMU (Atalet Ölçüm Birimi) sensörleri yer almaktadır.

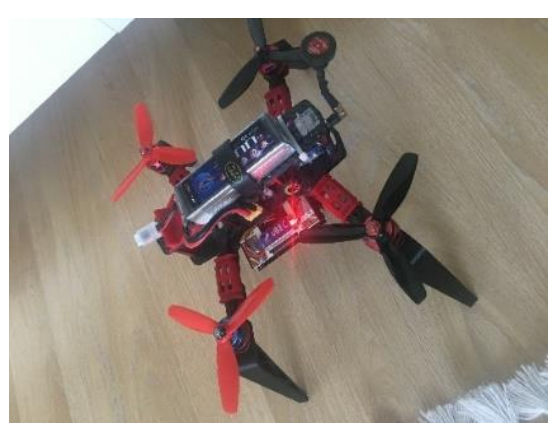

Şekil 2. Çalışmada kullanılan İHA

\subsection{Gömülü Sistem}

İHA üzerinde çalıştırılabilen gömülü sistemlerin geniş bir yelpazesi bulunmaktadır. Tercih edilecek gömülü sistemde derin öğrenme tekniği kullanılırken süreç boyunca verimliliğe ve planlamaya dikkat edilmelidir. Özellikle görüntü işleme kısmının optimize edilmesi ve çeşitli koşullar altında çalışan gömülü sistemde tek birimden görüntü işleme performansının sağlanması gerekmektedir. Bu amaçla İHA' lar için genellikle Raspberry, TinkerBoard ve Nvidia gömülü sistemler kullanılmaktadır. Bu sistemler içerisinde Nvidia'nın grafik işlem biriminin daha avantajlı olduğu görülmektedir. 
Bu çalışmada segmentasyon işlemlerini hızlandıran 4 çekirdekli ARM işlemcisine ve Maxwell görüntü kartına sahip olmasından dolayı Nvidia Jetson Nano gömülü sistem birimi tercih edilmiştir (Şekil 3). Bu gömülü sistem küçük yapısı gereği İHA' lara sabitlenebilmektedir.

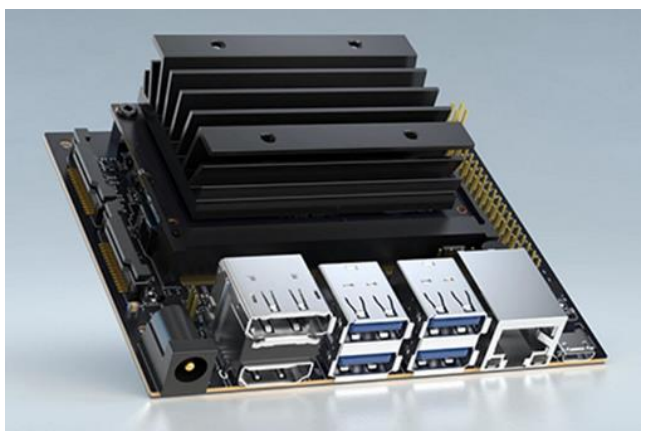

Şekil 3. Nvidia Jetson Nano [18]

NVIDIA'nın Jetson Nano geliştirme kiti platformu, bilgisayarlı görü modellerini verimli bir şekilde uygulamak için sinir ağ kütüphanelerini desteklemektedir. Bu sinir ağlarının kullanımını ise Grafik İşlem Biriminde (GPU) gerçekleştirmektedir [19]. Buna bağlı JetPack SDK yazılım ile uyumlu olması performans ve güç tüketimine katkı sağlamaktadır. Kart, özellikle 10 watt ve 5 watt olmak üzere iki güç modunu desteklemekte, her iki modda çeşitli CPU frekansları ve çekirdek sayısı için yapılandırılabilir özellikleri bulunmaktadır [20].

\subsection{Evrişimli Sinir Ağları}

Evrişimsel Sinir Ağı (Convolutional Neural Network, CNN), canlıların doğal görsel algılama mekanizmasından esinlenerek ortaya çıkarılmış derin öğrenme mimarisidir. 1990 yılında LeCun ve arkadaşları el yazısıyla rakamları sınıflandırabilen ve CNN'i oluşturan bir sinir ağı geliştirmiştir [21]. Evrişimli sinir ağlarındaki temel mantık görüntüyü ölçeklendirmek ve görselde nesnenin olup olmadığını denetlemektir. Şekil 4' te yer alan görselde, nesne denetimi gerçekleştirildikten sonra eğitilmiş bir kütüphane ile sınıflandırma işlemi görülmektedir [22].

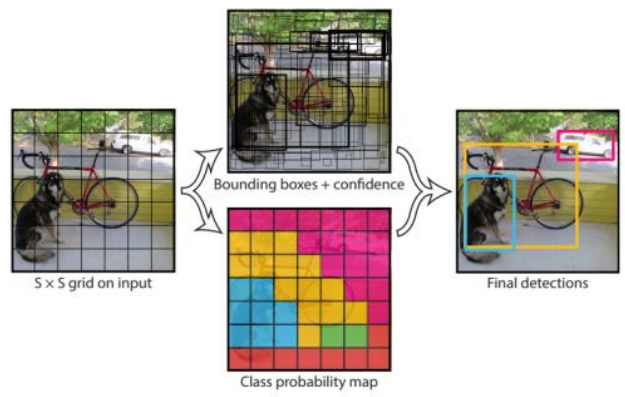

Şekil 3. Nesne Denetimi [23]

İHA içerisinde yer alan gömülü sistemler, yapay sinir ağlarını gerçek zamanlı çalıştırmaktadır. Buna bağlı olarak derin öğrenme yöntemlerinden hızlı sonuçlar alınmaktadır. Yakın zamana kadar, sinir ağlarındaki GPU gücüyle gerçek zamanlı çalışmanın mümkün olmadığı bilinmektedir.

Şekil 4'te İHA üzerinden alınan girdi örneğinin, kütüphanede bulunan eğitilmiş görsel veriler ile eşleştirilerek görüntüde sınıflandırma yapıldığı görülmektedir. Görüntü girdisindeki veriler gömülü sistem üzerinden işlenmektedir.

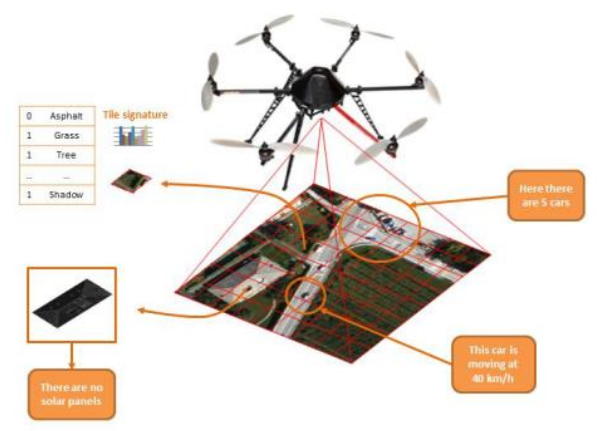

Şekil 4. İHA girdi örneği [24]

Genel olarak, derin öğrenme tabanlı nesne algılama algoritmaları iki aşamalı ve tek aşamalı nesne tespiti olarak iki sınıfa ayrılmaktadır. Tek aşamalı nesne tespitinde, nesne sınırlarını belirlemek için bir nesneden öznitelik çıkarılmaktadır. İki aşamalı nesne tespitinde ise, ağ her nesneden öznitelikler çıkarır, sınıflandırır ve sınır çizme işlemini gerçekleştirir. Bu amaçla çeşitli kütüphaneler kullanılarak yeni yöntemler geliştirilmektedir. Bu yöntemler yüksek doğruluk gösterse de, genellikle yüksek işlem gücü oluşturmaktadır.

Gömülü sistemde kullanılmak üzere AttentioNet, SSD, YOLO, DarkNet gibi çeşitli kütüphaneler bulunmaktadır. Bu yöntemler, gerçek zamanlı nesne algılama işlemlerinde hem işlemde zaman kazancı hem de ortalama hassasiyet doğruluğu (mAP - mean Average Precision) sağlamaktadır. Bu kütüphaneler CNN' ler ile kullanıldığında işlem hızını arttırmaktadır.

Şekil 3 'te verilen nesne denetimini formülize edecek olursak, nesne tespit çizgileri d'yi, yakalanan nesnedeki kabul edilen gerçek doğruluk g'yi ve nesne sayısı $K$ 'yı belirtmektedir. Birden fazla nesne tespiti olması durumunda vektör kümesi $(y)$, Eşitlik (1) ile ifade edilmektedir;

$$
y \mathrm{~d} \in\{1, \ldots, K\}
$$

Nesnenin tespit edildiği sınır çizgilerindeki sınıflandırılma $(\varphi)$ işlemi ise Eşitlik (2) ile formülize edilmektedir;

$$
\varphi(g \mathrm{~d} ; d)
$$

Tespit olmaması durumunda $\mathrm{d}$ negatif değer almaktadır. $\mathrm{Bu}$ durumda d sınır kutusu Eşitlik (3) 'te gösterilmiştir ( $\theta$ nesne parametrelerini temsil etmektedir). Sınıflandırma ise Eşitlik (4) ile gerçekleştirilmektedir.

$$
\begin{array}{r}
f \operatorname{loc}(I ; d, \theta) \\
f \operatorname{cls}(I ; d, \theta)
\end{array}
$$

Buna göre sınıflandırma formülündeki kayıplar Eşitlik (5)' de verilmiş olan formül üzerinden hesaplanabilmektedir.

$$
\begin{gathered}
\mathrm{L}(\mathrm{d}, \mathrm{I} ; \theta)=\alpha \operatorname{lloc}(\varphi(\mathrm{gd} ; \mathrm{d})-\text { floc }(\mathrm{I} ; \mathrm{d}, \theta)) \\
+\beta \operatorname{lcls}(\mathrm{yd}, \operatorname{fcls}(\mathrm{I} ; \mathrm{d}, \theta))
\end{gathered}
$$

Burada konum $(\alpha)$ ve siniflandirma $(\beta)$ kayıları ayarlanmaktadır. Bu kayıplar eklenerek nesnenin tespit edildiği verilerin ortalaması alınır ve model eğitilmek için $\theta$ (nesne parametreleri) en aza indirilmektedir [25].

\subsection{SSD MobileNet}

MobileNet SSD (Single Shot Multi Box Detector) modeli, içerisinde görsellerle öğretilmiş nesne sınıflandırmalarının 
bulunduğu modellerden biridir. SSD nesne detektörü olarak kullanılan popüler bir kütüphanedir. SSD MobileNet modelinin mimarisi, mobil ve gömülü tabanlı görsel uygulamalar için daha uygundur [26]. Yapılan araştırma sonucunda literatürde yaygın olarak kullanılan PASCAL VOC testleri Tablo 1'de paylaşılmıştır. Burada nesne tespiti metotları ve mAP (ortalama hassasiyet) birimi sonuçları verilmiştir [27].

Tablo 1. PASCAL VOC testleri [27]

\begin{tabular}{|c|c|c|}
\hline Metot & Data Test & $\begin{array}{c}\text { Ortalama } \\
\text { Hassasiyet }>\% 50 \\
(\mathrm{mAP})\end{array}$ \\
\hline $\begin{array}{c}\text { FAST R- } \\
\text { CNN }\end{array}$ & VOC $(07++12)$ & $\% 68,4$ \\
\hline $\begin{array}{c}\text { FASTER } \\
\text { R-CNN }\end{array}$ & $\begin{array}{c}\text { VOC } \\
(07++12++\mathrm{COCO})\end{array}$ & $\% 75,9$ \\
\hline YOLO & VOC $(07)$ & $\% 57,9$ \\
\hline SSD & $\begin{array}{c}\text { VOC } \\
(07++12++\mathrm{COCO})\end{array}$ & $\% 80$ \\
\hline
\end{tabular}

Tablo 1' e göre mAP ortalama hassasiyet verilerinde $\% 80$ başarı ile SSD metodunun önde olduğu görülmektedir. Şekil 5'te SSD MobileNet'in kavramsal olarak iki bölümden oluştuğu görülmektedir. İlk bölümde özellik haritaları üreterek nesne özellikleri oluşturulmakta, ikinci bölümde ise nesne tespitindeki sınırlayıcı çizgilerden, nesnenin konumu ve hareketi tahmin edilmektedir [26].

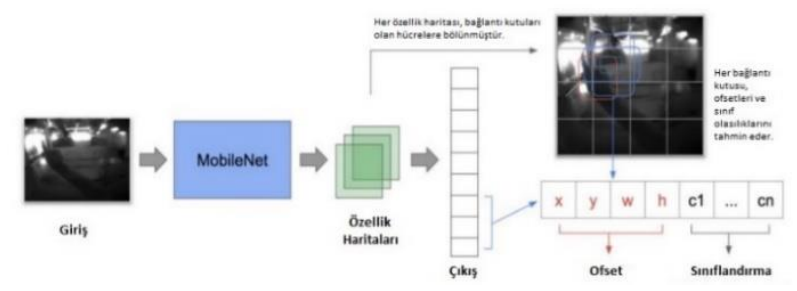

Şekil 5. SSD MobileNet [26]

Bu çalışmada SSD MobileNet'in görüntü boyutu parçalara bölünüp küçültülerek verinin tespitindeki hesaplama gücü azaltılmıştır. Önceden eğitilmiş bir ağda, küçük görüntü veri kümelerinin kullanılması derin öğrenme tekniği için çok yaygın ve güçlü bir yöntemdir.

\subsection{Uygulama}

İHA üzerinden nesne algılama işlemi, yüksek irtifadan alınan anlık görüntüler ile kütüphanede eşleşen görseller arasındaki benzerlik oranının belirlenmesi ile gerçekleştirilmiştir. Uygulamada nesnenin konumunun bulunması ve sınıflandırmasında Şekil 6' da verilen sabit odak modülüne sahip 1080p/30fps CSI Kamera kullanılmıştır.

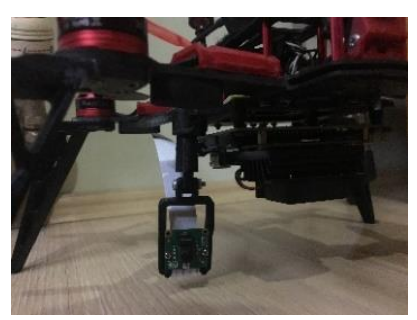

Şekil 6. CSI Kamera

Bu uygulamanın işleyişinde aşağıdaki adımlar izlenmektedir:

1. Kameradan görüntü alma işlemi

2. Görüntü gürültüleri giderme

e-ISSN: 2148-2683
3. Nesne belirleme ve sınır çizgisi oluşturma

4. Tespit edilen nesnenin sınırlayıcı çizgi ile takibi

5. Verilerin kaydedilmesi

Kameradan alınan görüntüler CSI bant aracılığı ile gömülü sisteme aktarılmıştır. Alınan görüntülerde uçuştaki titreşimden kaynaklanan parazitler gömülü sistem üzerinden ayrıştırılmıştır daha sonra nesne belirleme ve sınır çizgisi işlemi gerçekleştirilmiştir. Ardından SSD MobileNet aracılığı ile tespit edilen nesne hareketi sınırlayıcı çizgi ile takip edilmiş ve son olarak işlenmiş veriler kaydedilmiştir.

Gerçekleştirilen uygulamada tespit edilen nesneler, oluşturulan kare sınır çizgilerinde her nesne kategorisinin varlığı için yüzde cinsinden puanlar üretmektedir. Ek olarak, farklı çözünürlüklerdeki çok özellikli görüntülerden gelen tahminleri birleştirerek nesnelerin doğal olarak işlenmesini sağlamaktadır.

İHA' lar ile elde edilen görüntülerdeki zorluklardan biri bakış açısı varyasyonlarıdır. Gömülü Sistem, nesnenin farklı açılarından öğretilmiş özelliklerini kullanarak başarılı bir şekilde kütüphane karşılaştırması gerçekleştirmekte, Şekil 6' da görüldüğü gibi sınıflandırma işlemi yapmaktadır

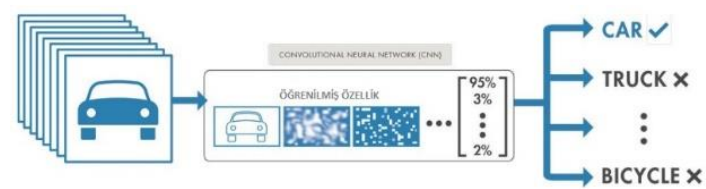

Şekil 6. Nesne Sinıflandırma [28]

$\mathrm{Bu}$ çalışmada kullanılan algoritmadaki derin öğrenme modelinde;

- İHA üzerinden alınmış gerçek zamanlı görüntüler, doğrudan öğrenilmiş veriler ile sentezlenmektedir.

- Veri kütüphanesinde bulunan görsel arşiv ile sonuç üretmektedir.

- Sınıflandırma işlemi çeşitli kütüphaneler ile yapilabilmektedir.

Algoritmanın gömülü sistem üzerinde işleyişi Şekil $7^{\prime}$ de gösterilmektedir. Kameradan alınmış görüntülerin gömülü sistem üzerinden sınır belirleme işlemi ile renkli sınır kutucukları oluşturulmaktadır. Belirlenen sınırlar içerisindeki özellikler, derin öğrenme kütüphanesindeki nesneler ile kıyaslanarak elde edilen değerler ekrana aktarılmaktadır.

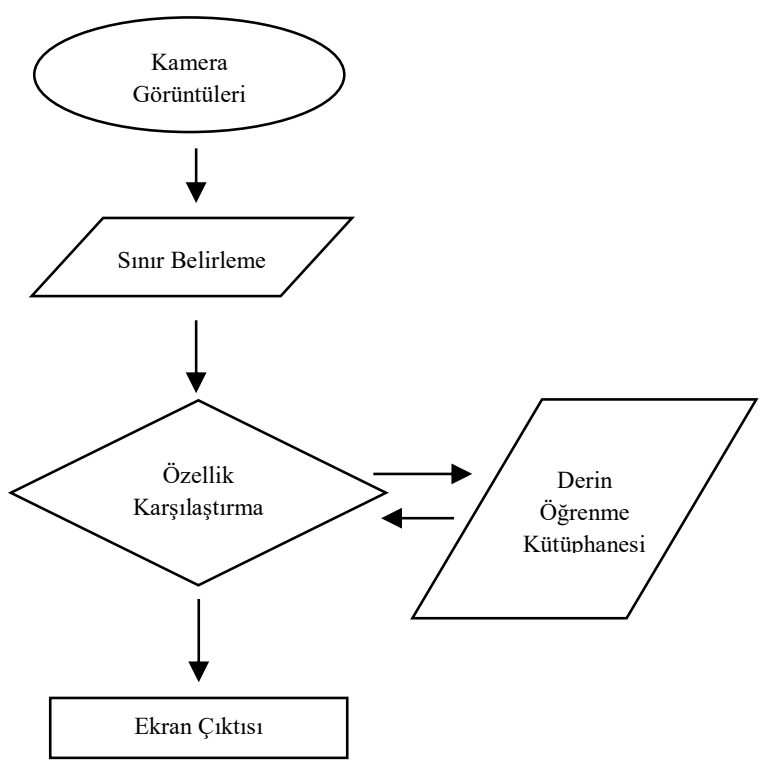

Şekil 7. Gömülü Sistem Algoritma Süreci 


\section{Araştırma Sonuçları ve Tartışma}

Bu çalışmada, İHA' larda Gömülü Sistem ile nesne belirleme işleminin yapılması amaçlanmıştır. Gömülü sistem seçilmesinde grafik işlem biriminin (GPU) olması sıradan mikroişlemcilere göre tercih sebebi olmuştur.

Algoritma tasarım öğeleri en aza indirilerek görüntülerin daha hızlı tepki vermesi sağlanmış, bunun yanında gömülü sistem tarafindan yönlendirilen algoritmaların çalışma ağırlı̆̆ını en aza indirmek, CPU kullanımını azaltmak, paralel işlemlerle işlem hızını artırmak için GPGPU (General Purpose Computation on Graphic Processing Units) tekniği kullanılmış ve grafik birimi üzerinden işlem yapılarak bellek yönetimi amaçlanmıştır. Böylece sistemin durmasının veya yavaşlamasının önüne geçilmiştir.

Çalışmada görüntüye giren nesnelerin ilk aldığı değerlere ilk kesinlik, nesnenin görüntüden çıktığı duruma ise son kesinlik denilmektedir. Şekil 8' de nesnenin görüntüden ayrıldı̆̆g görülmektedir. Burada değerlendirmeler mAP birimi üzerinden yapılmaktadır.

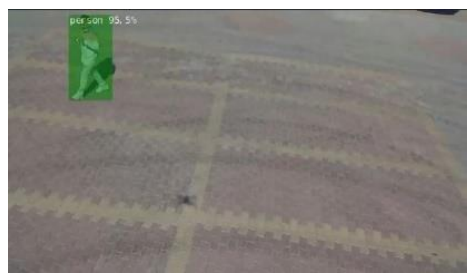

Şekil 8. İnsan tespitinde son kesinlik sonucu \%95.5 (mAP)

\section{1. İHA' dan Alınmış Görsel Veriler Üzerinden İnceleme}

Dört rotorlu İnsansız Hava Aracı üzerinden alınan görüntüler Nvidia Jetson Nano'ya bağlı kamera ile elde edilmiş ve anlık olarak işlenmiştir. Farklı ortamlardan alınan örnekler Şekil 9' da ve Şekil 10' da görülmektedir.

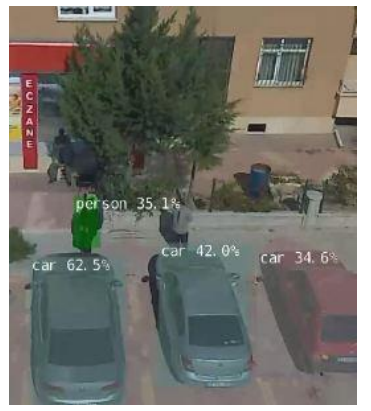

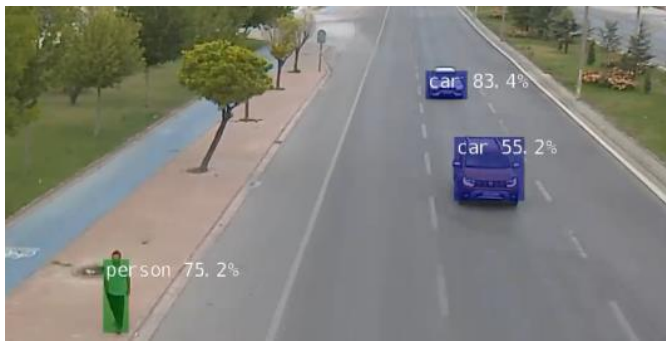

Şekil 10. İHA üzerinden alınan görüntü -2

Şekil 9 ve Şekil 10' da İHA üzerinde anlık olarak alınan verilerin işlenmesi sonucu nesne tespitinin sınıflandırma değerleri görülmektedir. Alınan verilerde irtifaya bağlı olarak farklı sonuçlar görülmektedir. Şekil 10'da \%75.2 mAP son kesinlik ile insan (person) sınıflandırıldığı görülmektedir.

$\mathrm{Bu}$ çalışmada elde edilen kayıtlar için, eşik değeri \%50’ den büyük olan ortalama hassasiyet (mAP) ölçütleri ile görüntüye giren nesnenin ilk algıladığ kesinlik değerleri, hareketli nesnenin görüntüden çıktığ 1 son kesinlik değerleri ve başarı durumları Tablo 2' de verilmiştir. Sonuç olarak, \%69.45 mAP ortalama hassasiyet ile insan, $\% 64.5 \mathrm{mAP}$ ortalama hassasiyet ile araç tespiti yapıldığı görülmektedir. Ortalama değerlerin böyle olmasına rağmen görüntüden çıkıncaya kadarki süreçte tespit edilen nesnelerin kesinliği önem arz etmektedir. Bu durum sınıflandırmaların (araç, insan, otobüs vb.) kesinliğinden daha emin olmamızı sağlamaktadır. Almış olduğumuz değerler ise insan için \%95.5 mAP son kesinlik ve araç için \% 83.4 mAP son kesinliktir.

Şekil 9. İHA üzerinden alınan görüntü -1

Tablo 2. Sinıflandırma ve başarı durum tablosu

\begin{tabular}{|c|c|c|c|c|}
\hline $\begin{array}{c}\text { Object } \\
\text { (Nesne) }\end{array}$ & $\begin{array}{c}\text { İk Kesinlik } \\
(\mathrm{mAP})\end{array}$ & $\begin{array}{c}\text { Son Kesinlik } \\
(\mathrm{mAP})\end{array}$ & $\begin{array}{c}\text { Ortalama Hassasiyet> \%50 } \\
(\mathrm{mAP})\end{array}$ & Başarı \\
\hline Car & $\% 45.6$ & $\mathbf{\% 8 3 . 4}$ & $\% 64.5$ & $\sqrt{ }$ \\
\hline Person & $\% 43.4$ & $\mathbf{\% 9 5 . 5}$ & $\% 69.45$ & $\sqrt{ }$ \\
\hline Bus & $\% 51.3$ & $\% 81.6$ & $\% 66.45$ & $\sqrt{ }$ \\
\hline Track & $\% 49.9$ & $\% 75.3$ & $\% 62.6$ & $\sqrt{ }$ \\
\hline
\end{tabular}




\section{Sonuç}

Hareketli nesne algılama, acil müdahale, kişi yoğunluğu, trafik yoğunlu ve sınır güvenliği gibi birçok uygulama tarafından tercih edilen önemli bir araştırma alanıdır. Bu çalışmada bir İHA ile geniş bir perspektiften derin örenme tabanındaki SSD MobileNet incelenmesi gerçekleştirilmiştir.

Literatürde yapılan bazı çalışmaların yöntem ve sonuçları Tablo 3'de verilmiştir.

Tablo 3. Literatürde yer alan nesne tespiti sonuçları

\begin{tabular}{|c|c|c|}
\hline Çalışma & Yöntem & $\begin{array}{c}\text { Sonuç } \\
(\mathrm{mAP})\end{array}$ \\
\hline$($ Piao, 2020) & YOLO & $\% 68.86$ \\
\hline$($ Yu, 2021) & DMNet & $\% 57.1$ \\
\hline$($ Donahue, 2014) & DeCAF & $\% 40.94$ \\
\hline (Ngiam,2019) & StarNet & $\% 66.88$ \\
\hline (Ouyang, 2015) & DNNs & $\% 50.7$ \\
\hline Bu Çalışmada & SSD MobileNet & $\mathbf{\% 6 9 . 4 5}$ \\
\hline
\end{tabular}

Tablo 3'de verilen sonuçlara göre ile literatürde yapılan çeşitli nesne tespiti uygulamalarına göre bu çalışmada daha yüksek ortalama mAP değeri elde edildiği görülmektedir.

\section{Kaynakça}

[1] Mukhamediev, R. I., Symagulov, A., Kuchin, Y., Zaitseva, E., Bekbotayeva, A., Yakunin, K., ... \& Tabynbaeva, L. (2021). Review of Some Applications of Unmanned Aerial Vehicles Technology in the Resource-Rich Country. Applied Sciences, 11(21), 10171.

[2] Liu, Y., Zhang, F., Huang, P., \& Zhang, X. (2021). Analysis, planning and control for cooperative transportation of tethered multi-rotor UAVs. Aerospace Science and Technology, 113, 106673.

[3] Hassler, S. C., \& Baysal-Gurel, F. (2019). Unmanned aircraft system (UAS) technology and applications in agriculture. Agronomy, 9(10), 618.

[4] Ong, G. Y. G. (2008, May). Electronic vehicle identification in the intelligent city. In IET Road Transport Information and Control-RTIC 2008 and ITS United Kingdom Members' Conference (pp. 1-6). IET.

[5] Volkova, L., Krisnawati, H., Adinugroho, W. C., Imanuddin, R., Qirom, M. A., Santosa, P. B., ... \& Weston, C. J. (2021). Identifying and addressing knowledge gaps for improving greenhouse gas emissions estimates from tropical peat forest fires. Science of the Total Environment, 763, 142933.

[6] Berrahal, S., Kim, J. H., Rekhis, S., Boudriga, N., Wilkins, D., \& Acevedo, J. (2016). Border surveillance monitoring using quadcopter UAV-aided wireless sensor networks. Journal of Communications Software and Systems, 12(1), 67-82.

[7] Tindall, J. A. (2006). Deconvolution of plant type (s) for homeland security enforcement using remote sensing on a uav collection platform. GEOLOGICAL SURVEY DENVER CO.

[8] SALUR, M. U., AYDIN, İ., \& Karaköse, M. (2019). gömülü derin öğrenme ile tehdit içeren nesnelerin gerçek zamanda tespiti. Dicle Üniversitesi Mühendislik Fakültesi Mühendislik Dergisi, 10(2), 497-509.

[9] Rokhmana, C. A. (2015). The potential of UAV-based remote sensing for supporting precision agriculture in Indonesia. Procedia Environmental Sciences, 24, 245-253.

[10] Demirhan, M., \& Premachandra, C. (2020). Development of an automated camera-based drone landing system. IEEE Access, 8, 202111-202121.

[11] Liu, M., Wang, X., Zhou, A., Fu, X., Ma, Y., \& Piao, C. (2020). Uav-yolo: Small object detection on unmanned aerial vehicle perspective. Sensors, 20(8), 2238.

[12] Yu, W., Yang, T., \& Chen, C. (2021). Towards resolving the challenge of long-tail distribution in UAV images for object detection. In Proceedings of the IEEE/CVF Winter Conference on Applications of Computer Vision (pp. 32583267).

[13] Donahue, J., Jia, Y., Vinyals, O., Hoffman, J., Zhang, N., Tzeng, E., \& Darrell, T. (2014, January). Decaf: A deep convolutional activation feature for generic visual recognition. In International conference on machine learning (pp. 647-655). PMLR.

[14] Ngiam, J., Caine, B., Han, W., Yang, B., Chai, Y., Sun, P., ... \& Vasudevan, V. (2019). Starnet: Targeted computation for object detection in point clouds. arXiv preprint arXiv:1908.11069.

[15] Ouyang, W., Wang, X., Zeng, X., Qiu, S., Luo, P., Tian, Y., ... \& Tang, X. (2015). Deepid-net: Deformable deep convolutional neural networks for object detection. In Proceedings of the IEEE conference on computer vision and pattern recognition (pp. 2403-2412).

[16] Petrova, T., \& Petrov, Z. (2020). Long Term Development Perspectives for UAV Potential. International E-Journal of Advances in Social Sciences, 6(16), 45-53.

[17] https://developer.nvidia.com/embedded/jetson-nano-dlinference-benchmarks, Jetson Nano: Deep Learning Inference. 03 Ocak 2022.

[18] https://developer.nvidia.com/embedded/jetson-developerkits, Jetson Nano. 03 Ocak 2022.

[19] Basulto-Lantsova, A., Padilla-Medina, J. A., Perez-Pinal, F. J., \& Barranco-Gutierrez, A. I. (2020, January). Performance comparative of OpenCV Template Matching method on Jetson TX2 and Jetson Nano developer kits. In 2020 10th Annual Computing and Communication Workshop and Conference (CCWC) (pp. 0812-0816). IEEE.

[20] Wang, X., \& Jia, K. (2020, July). Human fall detection algorithm based on YOLOv3. In 2020 IEEE 5th International Conference on Image, Vision and Computing (ICIVC) (pp. 50-54). IEEE.

[21] LeCun, Y., Boser, B., Denker, J., Henderson, D., Howard, R., Hubbard, W., \& Jackel, L. (1989). Handwritten digit recognition with a back-propagation network. Advances in neural information processing systems, 2 .

[22] Pastor Quiles, Y. (2018). Object detection and tracking using an UAV. 
[23] Singh, S., Suri, A., Singh, J. N., Singh, M., \& Yadav, D. K. (2021). Object Identification and Tracking Using YOLO Model: A CNN-Based Approach. In Machine Learning and Information Processing (pp. 153-160). Springer, Singapore.

[24] Moranduzzo, T. (2015). Detection and analysis methods for unmanned aerial vehicle images (Doctoral dissertation, University of Trento).

[25] Sun, C., Zhan, W., She, J., \& Zhang, Y. (2020). Object detection from the video taken by drone via convolutional neural networks. Mathematical Problems in Engineering, 2020.

[26] Pandey, R., White, M., Pidlypenskyi, P., Wang, X., \& Kaeser-Chen, C. (2017). Real-time egocentric gesture recognition on mobile head mounted displays. arXiv preprint arXiv:1712.04961.

[27] Liu, W., Anguelov, D., Erhan, D., Szegedy, C., Reed, S., Fu, C. Y., \& Berg, A. C. (2016, October). Ssd: Single shot multibox detector. In European conference on computer vision (pp. 21-37). Springer, Cham.

[28] https://www.mathworks.com/discovery/convolutionalneural-network-matlab.html. 03 Ocak 2022. 